\title{
Der Gesundheitsfonds: Neue Schieflagen - Keine Problemlösung
}

Am 1. Januar 2009 tritt bekanntlich der Gesundheitsfonds in Kraft. Er stellt zwar einen neuen organisatorisch-institutionellen Rahmen für die Finanzierung der gesetzlichen Krankenversicherung (GKV) dar, nimmt aber, sieht man einmal von der Einführung eines steuerfinanzierten Bundeszuschusses ab, keine grundlegenden Veränderungen am bisherigen Finanzierungsmodus vor: Von einer Auf- oder substanziellen Anhebung der Versicherungspflichtgrenze oder der Beitragsbemessungsgrenze sieht er ebenso ab wie von einer Einbeziehung anderer Einkunftsarten in die Beitragsbemessung.

Dennoch ist bereits heute absehbar, dass der Gesundheitsfonds dazu beitragen wird, die Finanzierungslasten in der GKV mittelfristig weiter von den Arbeitgebern auf die Versicherten zu verlagern. Wenn eine Krankenkasse ihre Ausgaben mit den ihr zugewiesenen Mitteln nicht mehr decken kann, muss sie entweder die kassenspezifischen Leistungen kürzen oder einen Zusatzbeitrag erheben, der allein von den Versicherten - und nicht vom Arbeitgeber - aufgebracht wird. Die Summe aller Zusatzbeiträge kann auf bis zu $5 \%$ der GKV-Gesamtausgaben steigen - der Deckungsgrad durch den Gesundheitsfonds also auf $95 \%$ sinken. Erst wenn dieser Wert überschritten wird, ist eine Anhebung des gemeinsam von Versicherten und Arbeitgebern finanzierten Beitragssatzes vorgesehen. Man darf davon ausgehen, dass sich alsbald eine neue Kluft zwischen Einnahmen und Ausgaben in der Gesetzlichen Krankenversicherung auftun wird. De facto wird mit der Konstruktion des Zusatzbeitrags die alte Forderung von Union und Arbeitgebern nach einem Einfrieren des Arbeitgeberanteils an den GKV-Beiträgen erfüllt. Zudem steht in den Sternen, ob die Bundesregierung tatsächlich den Beitragssatz anhebt, wenn die Gesamtsumme der Zusatzbeiträge die 5-\%Schwelle erreicht haben wird. Denn es ist ein Leichtes, den gesetzlich vorgeschriebenen Deckungsanteil des Gesundheitsfonds beim Erreichen des Schwellenwerts von $95 \%$ weiter zu senken und den der Zusatzbeiträge zu erhöhen. Es bedarf jedenfalls keiner blühenden Phantasie, um sich vorzustellen, dass in diesem Fall das Standortargument für die Forderung bemüht werden wird, das Finanzierungsdefizit nicht durch eine Anhebung des Beitragssatzes, sondern der Zusatzbeiträge zu decken.

Die Festsetzung eines bundeseinheitlichen Beitragssatzes bedeutet, dass es unter den Versicherten Gewinner und Verlierer gibt, denn bisher klaffen die Beitragssätze der Krankenkassen erheblich auseinander. Gegen einen einheitlichen Beitragssatz ist aus der Perspektive einer solidarischen Krankenversicherung nichts einzuwenden, denn diejenigen, die bisher noch in einer günstigeren Kasse versichert sind, profitieren folglich davon, dass der Gesundheitszustand ihrer jeweiligen Versichertengemeinschaft überdurchschnittlich gut und der Behandlungsbedarf daher vergleichsweise gering ist. Allerdings wird die Ungleichheit in der GKV-Finanzierung recht bald über den kassenindividuellen Zusatzbeitrag zurückkehren. Dass der Beitragssatz ab 1.1.2009 mit 15,5\% erheblich über dem gegenwärtigen durchschnittlichen allgemeinen Beitragssatz liegt, hat nichts mit der Einführung des Gesundheitsfonds zu tun, sondern ist aufden Anstieg der Ausgaben für ambulante ärztliche Behandlung, für Krankenhausbehandlung und für Arzneimittel zurückzuführen.

Außerdem ist erstmals die direkte Berücksichtigung von Erkrankungen bei der Finanzmittelzuweisung an die Kassen vorgesehen. Dieser „morbiditätsorientierte Risikostrukturausgleich“ (MorbiRSA) tritt gemeinsam mit dem Gesundheitsfonds in Kraft. Allerdings gleicht er nur einen Teil der krankheitsbedingten Ausgabenrisiken der Krankenkassen aus, denn das GKV-Wettbewerbsstärkungsgesetz beschränkt ihn auf maximal 80 Krankheiten, für deren Behandlung die Krankenkassen pro Kopf mindestens $50 \%$ mehr als im Durchschnitt für ihre Versicherten aufwenden. Die Finanzsituation der Krankenkassen mit einem überdurchschnittlichen Anteil chronisch Kranker wird sich also gegenüber den Konkurrenten etwas verbessern, aber sie sind weiterhin strukturell benachteiligt. Sie werden daher gezwungen sein, den erwähnten Zusatzbeitrag früher zu erheben und stärker anzuheben als andere.

Der Gesundheitsfonds trägt also nichts zur Lösung der Finanzierungsprobleme in der GKV bei: Weder stell ter die Finanzierung der GKV auf eine dauerhaft tragfähige Grundlage noch beseitigt er die Gerechtigkeitsdefizite in der Finanzierung der Gesetzlichen Krankenversicherung. Vielmehr beinhaltet er mittelfristig eine weitere Verlagerung der Finanzierungslasten von den Arbeitgebern auf die Versicherten. Zwar ist die Einführung von Elementen eines morbiditätsorientierten Risikostrukturausgleichs im Ansatz ein Fortschritt; dessen Konstruktion bleibt allerdings unzureichend, und auch nach seiner Einführung werden starke Anreize zur Risikoselektion fortbestehen. Die Konstruktion des Zusatzbeitrags und die nur partielle Berücksichtigung der Morbidität bei der Finanzmittelzuweisung werden die Krankenkassen mit einem hohen Anteil an einkommensschwachen und chronisch kranken Mitgliedern weiterhin strukturell benachteiligen.

Weitere Grundsatzentscheidungen über die Finanzierung der GKV sind auf die kommende Legislaturperiode verschoben. Selbstverständlich hängt die Richtung, die dann eingeschlagen werden wird, stark von den künftigen politischen Mehrheitsverhältnissen ab.

\footnotetext{
Professor Dr. Dr. Thomas Gerlinger, Professor für Medizinische Soziologie an der Goethe-Universität Frankfurt/M. Arbeitsschwerpunkte: Gesundheitssystemforschung, internationaler Verg/eich von Gesundheitssystemen, Gesundheitspolitik. e-mail: Gerlinger@em.uni-frankfurt.de
} 